\title{
Droit d'asile en France : état des lieux (partie3)
}

\section{(2) OpenEdition \\ Journals}

Édition électronique

URL : http://journals.openedition.org/conflits/614

DOI : $10.4000 /$ conflits.614

ISSN : $1777-5345$

Éditeur :

CCLS - Centre d'études sur les conflits lilberté et sécurité, L'Harmattan

Édition imprimée

Date de publication : 15 octobre 1996

ISSN : 1157-996X

\section{Référence électronique}

"Droit d'asile en France : état des lieux (partie3) », Cultures \& Conflits [En ligne], 23 | automne 1996, mis en ligne le 15 mars 2006, consulté le 30 mars 2021. URL : http://journals.openedition.org/conflits/614 ; DOI : https://doi.org/10.4000/conflits.614

Ce document a été généré automatiquement le 30 mars 2021

Creative Commons License 


\section{Droit d'asile en France : état des lieux (partie3)}

1 Une procédure " prioritaire "

2 L'utilisation aléatoire de l'article 31 bis 3 et $4^{1}$ Le préfet peut refuser l'admission au séjour d'un demandeur d'asile notamment en cas de menace grave pour l'ordre public (article 31 bis 3), de fraude délibérée, de recours abusif ${ }^{2}$ ou de demande présentée uniquement en vue de faire échec à une mesure d'éloignement (article 31 bis 4). Cette disposition comporte par essence le risque d'une appréciation du bien-fondé d'une demande de protection par la préfecture alors que ce n'est pas son rôle mais celui de l'O.F.P.R.A. En 1994, 516 demandes ont été ainsi traitées, 620 en 1995 et 581 en $1996^{3}$.

3 Lorsqu'un étranger est contrôlé en situation irrégulière et qu'il demande alors l'asile, la préfecture déduit trop souvent de cette irrégularité que sa demande est sans fondement. Le nombre de convocations délivrées par l'O.F.P.R.A. à des demandeurs du statut de réfugié dans le cadre de la procédure prioritaire constitue une indication de l'application abusive de l'article 31 bis $^{4}$. La circulaire du 8 février 1994 précise pourtant clairement qu'une demande ne peut être considérée comme présentée en vue de faire échec à une mesure d'éloignement que si elle est " manifestement dilatoire, formée dans le seul but " d'y échapper, que s'il ne subsiste aucun " doute sur l'absence de réalité des craintes alléguées " et que " s'il apparait que les raisons invoquées par l'intéressé pour justifier le retard à se présenter spontanément pour demander l'asile sont dénuées de fondement ${ }^{15}$. L'application de cette procédure est particulièrement abusive lorsqu'elle touche des personnes contrôlées à proximité de la frontière, cellesci n'ayant pas encore eu le temps matériel de se rendre à la préfecture.

Des ressortissants du Kosovo qui cherchaient à se rendre en Allemagne et qui ont été remis par les autorités allemandes aux autorités françaises - au motif qu'ils avaient traversé la France auparavant - se sont vu immédiatement appliquer cet article. En octobre 1996, ce genre d'abus a touché des Kurdes irakiens qui, après avoir été interpellés à Nice, ont fait l'objet de reconduites à la frontière. À la même date, après avoir cherché à déposer une demande d'asile, un ressortissant tchadien s'est vu appliquer l'article 31 bis 4 au motif que sa demande constituait " un recours abusif aux procédures d'asile, uniquement destinée à lui permettre de rester en France, à 
l'expiration de son visa touristique ". Pourtant, aucun élément de fait ne semblait légitimer une telle décision. Aucune mesure d'éloignement n'avait été prise à son encontre, son visa étant encore valable 7 semaines, et c'était la première fois qu'il déposait une demande d'asile ${ }^{6}$.

5 Dans l'affaire de Sospel ${ }^{7}$, le Conseil d'État a annulé, pour excès de pouvoir, dix-huit arrêtés de reconduite à la frontière, qui avaient été pris à l'égard de Tziganes venus d'ex-Yougoslavie, en considérant qu'il ne ressortait pas " des pièces du dossier que cette admission pouvait être légalement refusée à ces derniers pour l'un des motifs limitativement énumérés par les dispositions des 1 à 4 de l'article 31 bis de l'ordonnance du 2 novembre 1945 "8.

Cet abus de procédure peut être également dénoncé dans le cadre de l'application de l'article 31 bis 3 : un Pakistanais refoulé de Grande-Bretagne a été considéré comme représentant une menace pour l'ordre public car il " avait précédemment séjourné clandestinement en France et avait usé d'un faux passeport néerlandais "9! Cette appréciation est d'autant plus abusive que la circulaire du 8 février 1994 prévoit que " l'autorisation provisoire de séjour ne peut être refusée que si sa présence en France constitue une menace grave pour l'ordre public ".

7 Un examen " prioritaire " par l'O.F.P.R.A. et un recours non suspensif

8 Dans les cas visés aux alinéas 3 et 4 de l'article 31 bis, le préfet peut prendre sans délai un arrêté préfectoral de reconduite à la frontière ${ }^{10}$ " qui ne peut être mis à exécution avant la décision de l'Office "11.

9 Le demandeur doit remplir son dossier de demande de reconnaissance du statut de réfugié " immédiatement "12. Il est en général maintenu en rétention administrative et il lui sera difficile de comprendre les subtilités de la procédure, de rassembler les documents nécessaires, de trouver un conseil de son choix et un interprète pour préparer son dossier de manière attentive ${ }^{13}$. Il se trouve en outre partagé entre cette rédaction et la préparation d'un éventuel recours contre l'arrêté de reconduite à la frontière qui doit être déposé devant le tribunal administratif dans les 24 heures suivant la notification ${ }^{14}$.

10 L'article 2 alinéa 5 de la loi du 25 août 1952 modifiée en 1993 précise que " l'O.F.P.R.A. statue par priorité " et la circulaire du 8 février 1994 prévoit que " sa décision puisse intervenir dans de brefs délais compatibles avec les délais de rétention administrative ". Cet examen par priorité se déroule dans des conditions peu satisfaisantes au regard de l'enjeu de la démarche, avec de probables difficultés d'interprétariat pour nombre de ces dossiers. En général, la demande consiste en un court récit adressé par télécopie.

11 On peut se demander si cette procédure est en conformité avec le principe de " confidentialité des éléments d'information " réservés à l'O.F.P.R.A. : ce principe, dégagé par le Conseil constitutionnel dans sa décision du 22 avril 1997, constitue une " garantie essentielle du droit d'asile " et implique " que seuls les agents habilités à mettre en œuvre le droit d'asile, notamment par l'octroi du statut de réfugié, peuvent avoir accès à ces informations ". Dans sa décision, le Conseil constitutionnel a condamné " la possibilité donnée à des agents des services du ministère de l'Intérieur et de la gendarmerie nationale d'accéder aux empreintes digitales des demandeurs du statut de réfugié ". En l'occurrence, la procédure prioritaire oblige le demandeur à confier à la police le texte de sa demande de reconnaissance de statut de réfugié sans aucune garantie de confidentialité. 
12 Au niveau de l'O.F.P.R.A., il est regrettable qu'un examen particulièrement approfondi ne soit pas exigé, le délai moyen d'instruction étant de soixante-douze heures ${ }^{15}$, et que des entretiens ne soient pas prévus systématiquement. Ce souci de vigilance se trouve pourtant dans la circulaire de 1994 qui prévoit que " l'examen par l'O.F.P.R.A. de ces demandes bénéficie des mêmes garanties que celles accordées à toute demande " et envisage " des investigations particulières, des auditions et des compléments d'information ". Le H.C.R. a recommandé que l'Office puisse " disposer des moyens nécessaires pour procéder à l'audition des demandeurs "16 se trouvant sous le coup de l'article 31 bis, en insistant sur la situation particulière des ressortissants d'Algérie, d'ex-Yougoslavie ou des Kurdes de Turquie. Le Conseil de l'O.F.P.R.A. a ensuite confirmé qu'il est " souhaitable d'organiser des missions pour procéder à leur audition en province ".

13 Lorsque l'O.F.P.R.A. pense qu'une demande requiert un examen plus complet, il ne s'estime pas lié par les délais impartis par les préfectures et traite cette demande selon la procédure normale. Les demandeurs ne se voient généralement pas délivrer une autorisation provisoire de séjour (A.P.S.), mais sont mis sous régime de convocation ou assignés à résidence ${ }^{17}$. Lorsque les étrangers ne peuvent plus être maintenus en rétention, ils sont mis en liberté. Si l'O.F.P.R.A. veut les convoquer, il n'est pas sûr que la convocation leur parvienne. S'ils se trouvent en province, leur acheminement ne sera pas prévu. Ainsi, il arrive souvent qu'ils ne se rendent pas à une convocation de l'O.F.P.R.A., ce qui n'empêche pas ce dernier de statuer, en général négativement.

L'étranger dont l'office a rejeté la demande en " procédure prioritaire " peut être éloigné immédiatement, le recours devant la Commission des recours des réfugiés perdant alors son caractère suspensif.

Les demandeurs d'asile " admis au séjour "

16 Dans leur majorité, les demandeurs d'asile sont admis au séjour. Le décret du 2 septembre 1994 prévoit que le demandeur doit se présenter à la préfecture pour retirer le formulaire O.F.P.R.A.; il reçoit alors une A.P.S. d'un mois ${ }^{18}$. Après réception du dossier par l'office, un récépissé de demande d'asile valant A.P.S., de 3 mois renouvelables, est remis jusqu'à la notification de la décision de la Commission des recours des réfugiés. Depuis 1991, les demandeurs du statut de réfugié n'ont plus le droit de travailler sous prétexte d'une réduction des délais d'instruction de leur demande. La durée moyenne de la procédure, de la formulation de la demande à la décision de la Commission, est de six à huit mois, dont environ quatre mois pour la Commission, mais elle peut parfois atteindre plusieurs années. Quelques milliers de demandeurs bénéficient d'un hébergement en Centre d'accueil pour les demandeurs d'asile (C.A.D.A.), les autres reçoivent une allocation d'insertion de $1300 \mathrm{~F}$ par mois pendant une durée maximum d'une année. Cette allocation n'a pas été réévaluée depuis plus de 10 ans.

17 L'asile dit " territorial "

18 Les instruments juridiques internationaux en matière de réfugiés ne prévoient une protection que pour ceux qui sont considérés comme entrant dans le cadre de la définition de réfugié telle qu'établie par la Convention de Genève relative au statut des réfugiés de 1951. Aucun texte international ou européen n'institue une autre forme de protection. 
Certains États ont mis en place un système afin de permettre le séjour des personnes qui courent un risque de persécution en cas de retour dans leur pays mais qui soit ne désirent pas demander le statut de réfugié, soit ne l'ont pas obtenu, n'ayant pas été autorisées à le solliciter ou ayant vu leur demande refusée ${ }^{19}$. Les conditions posées à l'accès et à l'octroi de ce type de protection ainsi que l'étendue des droits attribués aux bénéficiaires diffèrent. Dans plusieurs pays comme la France, cet asile relève de la pratique administrative, dans d'autres, comme les Pays-Bas ou la Grande-Bretagne, une base juridique peut exister en droit interne.

Cette forme d'asile est soumise à la volonté souveraine de l'État et consiste, d'une manière générale, en un titre de séjour n'offrant souvent aucune garantie de droits, de procédure, ni de durée, contrairement à la reconnaissance du statut de réfugié. C'est pourquoi il convient de ne pas se satisfaire de son extension par défaut aux personnes faisant l'objet d'une interprétation restrictive de la Convention de Genève. En France, le nombre de personnes bénéficiant de l'asile territorial est difficile à connaître. Diverses mesures ont concerné des ressortissants de Pologne ou du Liban dans les années quatre-vingt puis de l'ex-Yougoslavie à partir de 1992. Aujourd'hui, le cas typique est celui des Algériens menacés par des groupes armés islamistes.

\section{Un statut précaire ou le caractère discrétionnaire de la protection}

En France, la protection offerte par l'asile territorial est accordée discrétionnairement et résulte essentiellement de circulaires, de télégrammes, voire d'instructions, généralement non publiés afin de ne pas créer $d$ '" effet d'appel ". Ce sont des textes internes à l'administration dont les bénéficiaires ne peuvent se prévaloir. Ainsi, l'État se donne la possibilité de lever unilatéralement la protection s'il estime que le risque en cas de retour a disparu ${ }^{20}$.

La protection varie d'un groupe de bénéficiaires à l'autre, voire au sein d'une même nationalité : par exemple, pour les Algériens, il n'existe qu'un seul texte connu laissant place à une large appréciation et déterminant un examen au cas par cas, alors que, pour les ex-Yougoslaves, les textes sont nombreux, détaillés et d'ordre plus général. Il existe des situations comparables, où l'État se contente de suspendre les mesures d'éloignement sans admettre les personnes au séjour ${ }^{21}$.

\section{L'exemple des Algériens}

Selon les années, de $1 \%$ à $4 \%$ des Algériens qui demandent le statut de réfugié $l^{\prime}$ obtiennent ${ }^{22}$. Ceux qui invoquent des menaces de la part des mouvements islamistes se voient presque tous écartés de la protection de la Convention de Genève du fait d'une jurisprudence restrictive critiquée par le H.C.R. ${ }^{23}$. Dans une note concernant les Algériens, le H.C.R. a rappelé en janvier 1995 que " les demandeurs qui affirment de façon crédible être menacés par des militants de groupes islamistes devraient normalement obtenir le statut de réfugié" et a ajouté que " les personnes susceptibles d'obtenir le statut mais qui ne se le verraient pas accorder [...] devraient être autorisées à bénéficier de la protection qu'elles requièrent tout comme des droits socioéconomiques élémentaires, de préférence au moyen de l'octroi d'un statut alternatif clairement défini ".

Le seul texte qui règle la situation de ces Algériens est un simple télégramme de quelques lignes, non publié, de la Direction des libertés publiques et des affaires juridiques du ministère de l'Intérieur (D.L.P.A.J.), en date du 22 décembre 1993. Avant d'accorder une autorisation provisoire de séjour ou un titre de séjour à une personne " 
arguant de menaces ou de risques pour sa vie, sa liberté ou sa sécurité en Algérie du fait des activités des groupes islamistes ", les préfets doivent saisir la D.L.P.A.J. à l'appui d'un rapport concernant l'intéressé devant contenir " outre les éléments habituels, notamment les points suivants : nature des études effectuées ou en cours, [...] présence en France d'une famille proche, [...] ressources disponibles et logement ".

L'instruction du dossier dure souvent plusieurs mois et peut aller jusqu'à quinze mois. En attendant, les demandeurs sont en général maintenus au séjour sous couvert d'une simple convocation à la préfecture qui ne leur permet pas de travailler. Les préfectures sont invitées à saisir une commission interministérielle (Intérieur, Affaires étrangères, Affaires sociales) qui décide au cas par cas selon des critères non déterminés et sans motivation des refus. Selon le compte rendu de la réunion du Conseil de l'O.F.P.R.A. du 29 mai 1995, les cas sérieux de menaces avérées peuvent être signalés par l'Office à l'attention de la D.L.P.A.J. en vue de " rechercher une solution dans une formule d'asile territorial "24.

Depuis janvier 1994, environ 2500 Algériens auraient bénéficié d'un tel asile territorial. Dans la pratique, il apparaît que seuls les Algériens qui sont entrés en France régulièrement se voient remettre un titre de séjour ${ }^{25}$. La solution demeure souvent provisoire, le titre obtenu étant valable pour une durée de trois ou six mois renouvelables. Ils peuvent obtenir une autorisation provisoire de travail à condition de produire une promesse d'embauche. L'entreprise s'avère difficile lorsque l'A.P.S. indique que le titulaire devra quitter le territoire à l'issue du séjour autorisé et que l'" autorisation ne permet pas d'occuper un emploi ", ce qui est particulièrement dissuasif pour un éventuel employeur. Par ailleurs, il convient de préciser que lorsque l'étranger obtient une autorisation de travailler, celle-ci peut n'être valable qu'à l'égard de l'emploi pour lequel elle a été obtenue. Le droit au regroupement familial n'existe pas et il n'y a aucune certitude quant au renouvellement du titre de séjour.

La circulaire du 24 juin 1997 du ministère de l'Intérieur devrait permettre de résoudre certaines de ces situations. En effet, en ce qui concerne les Algériens qui s'estimeraient menacés en cas de retour, les dossiers doivent continuer à être transmis à la D.L.P.A.J. et les personnes qui sont admises au bénéfice de l'asile territorial devraient se voir remettre un certificat de résidence d'un an après un premier délai de six mois. ministère de l'Intérieur du 3 août 1992 a garanti le maintien des ressortissants yougoslaves sur le territoire " en raison de la persistance d'une situation troublée dans plusieurs territoires de l'ex-Yougoslavie " et a demandé aux préfets " de ne pas mettre à exécution les arrêtés de reconduite à la frontière pris à l'encontre des ressortissants de l'ex-Yougoslavie et de ne pas en prendre de nouveaux "26. Il prévoyait en outre la délivrance d'autorisations de séjour (A.P.S.) renouvelables en fonction de l'évolution de la situation en ex-Yougoslavie. Un autre télégramme du ministère de l'Intérieur, du 8 février 1993, traitait de l'accès à certaines prestations sociales et la circulaire du 23 janvier 1990 prévoyait la délivrance d'une autorisation de travail sous certaines conditions.

32 L'obligation de visa restait en vigueur sur le plan des principes. Le télégramme du 3 août 1992 reconnaissait cependant " la difficulté d'accéder aux consulats de France " et déclarait que les personnes arrivées sans visa " se verront délivrer une A.P.S. de 3 mois " (renouvelable) mais seulement si la région dont elles sont originaires est troublée ${ }^{27}$ et 
si elles n'ont pas " durablement transité dans des États tiers ". Le télégramme du 8 février 1993 a étendu le bénéfice des A.P.S. d'une durée de 6 mois " aux personnes entrées en France sous couvert d'un visa délivré par notre consul à Zaghreb ".

Ceux qui étaient en possession d'une promesse d'embauche ou d'un contrat de travail pouvaient obtenir une autorisation provisoire de travail ayant la même durée que l'A.P.S. ${ }^{28}$.

En 1993, le H.C.R. ${ }^{29}$ a recommandé qu'en cas de prolongation du séjour le regroupement familial soit permis pour les conjoints, les enfants mineurs et toute autre personne dépendante des bénéficiaires de la protection temporaire. Cette demande est conforme à l'article 8 de la Convention européenne des droits de l'Homme. En France, aucun dispositif n'a été prévu pour les titulaires d'A.P.S. et il est resté difficile d'obtenir des visas touristiques pour venir rejoindre en France des membres de la famille. Le ministère de l'Intérieur ${ }^{30}$ a seulement précisé que des visas pouvaient être accordés " pour tenir compte de situations particulières, principalement le rapprochement familial entre personnes déplacées ".

La levée de la protection temporaire

Environ un million de Bosniaques se sont rendus dans les différents pays européens, la plupart bénéficiant d'une protection temporaire. Ces États se sont interrogés, après la conclusion des Accords de Dayton, sur l'opportunité de prolonger cette protection. L'annexe 7 de ces Accords, portant sur les réfugiés et les personnes déplacées, a confié au H.C.R. la tâche de mettre au point un plan de rapatriement en consultation avec les parties signataires et les États d'accueil.

Conformément à son mandat, le H.C.R. a préconisé ${ }^{31}$ de ne pas précipiter le renvoi des populations bosniaques tant que la garantie des droits de l'homme et la sécurité ${ }^{32}$ ne seront pas effectivement assurées. Vu l'évolution de la situation, Madame Ogata, Haut Commissaire des Nations Unies pour les Réfugiés, a déclaré le 17 juin 1996 " qu'elle n'était toujours pas en position de recommander la levée de la protection temporaire pour les centaines de milliers de réfugiés bosniaques en Europe ". Il était ainsi fortement suggéré par le H.C.R., d'une part, que les États d'accueil maintiennent la protection temporaire ${ }^{33}$ et, d'autre part, qu'ils autorisent le recours au " retour exploratoire ". Pour permettre les retours volontaires, à titre exploratoire ou à titre définitif, le H.C.R. a publié des Repatriation Information Reports dans lesquels il donnait la liste de zones " possibles de retour " (sous réserve des conditions d'hébergement et des infrastructures en place).

En France, la circulaire du 13 juin 1996 du ministère de l'Intérieur a organisé les modalités de ce voyage exploratoire pour les ex-Yougoslaves (quelle que soit leur nationalité) réfugiés ou bénéficiant d'une mesure de protection temporaire. Il s'agissait de permettre " aux personnes qui le souhaitent " de se rendre à l'endroit de leur choix ${ }^{34}$ afin qu'elles " puissent décider, en toute connaissance de cause, de leur réinstallation dans leur pays d'origine ". Les réfugiés statutaires ont été autorisés à utiliser, à titre exceptionnel, leur " titre de voyage pour réfugié ". Pour les personnes bénéficiant de la protection temporaire, les préfectures pouvaient apposer un visa préfectoral de retour sur leurs documents de voyage.

Une seconde circulaire, du 28 juin 1996, contient la lettre à transmettre à ces ressortissants afin de les informer sur les possibilités de retour et les aides accordées en 
cas de retour définitif. En revanche, il n'y a pas d'aide officielle prévue pour faciliter le voyage exploratoire.

Par ailleurs, la circulaire du 3 août 1992 précise que les bénéficiaires de l'asile territorial " conservent la possibilité d'introduire une demande de reconnaissance du statut de réfugié ". Avant tout renvoi, ces personnes devraienteffectivement pouvoir solliciter la reconnaissance du statut de réfugié. Hervé de Charette, alors ministre des Affaires étrangères, a déclaré, dans une lettre du 31 janvier 1996, " que les couples mixtes ou les personnes issues de mariages mixtes ${ }^{35}$ auront accès à la procédure de détermination du statut de réfugié et bénéficieront d'un examen équitable et approfondi de leurs demandes éventuelles ". 
Convention de Genève ". L'article 3 précise que le directeur est nommé par le ministre des Affaires étrangères pour une durée de trois ans.

51 Il serait exagéré d'affirmer que le ministère des Affaires étrangères adresse des instructions générales à l'O.F.P.R.A. concernant le traitement des dossiers. Cependant, les contacts qui ont lieu avec les services ministériels intéressés peuvent amener à un rapprochement des analyses. Selon un document de l'office, le représentant du ministre des Affaires étrangères qui préside le Conseil de l'Office " joue un rôle éminent dans la définition de l'orientation générale de l'activité de l'office et des règles générales concernant l'admission à la qualité de réfugié ". Ce document ajoute que, " outre son rôle traditionnel d'interprétation des traités, le ministère est l'une des sources d'information sur la situation dans les pays d'origine ou même sur des cas particuliers ". En effet, les ambassades de France à l'étranger sont régulièrement consultées pour le traitement individuel de certains dossiers.

Dans certains cas, il semble évident que des conseils pressants sont donnés par le gouvernement: ainsi, une centaine de dossiers de Tunisiens, la plupart semble-t-il, membres, sympathisants ou supposés tels du parti islamiste Ennahda (Renaissance), sont restés " gelés ", certains depuis cinq ans. Même ceux qui avaient déjà été reconnus réfugiés par le H.C.R. lors d'un séjour provisoire en Algérie ont dû attendre des années pour se voir confirmer cette décision par l'office.

\section{L'harmonisation en Europe}

Les États membres de l'Union européenne ont entamé des travaux d'harmonisation afin, notamment, de rapprocher leurs positions. En 1995, ils ont adopté la Position commune concernant l'application harmonisée de la définition de réfugié de la Convention de Genève. Au sujet des victimes d'exactions commises par des agents extérieurs à l'État, le H.C.R. s'est dit préoccupé par l'autorisation donnée aux États membres de ne pas leur reconnaître le statut de réfugié en ajoutant que cela était " contraire au texte et à l'esprit de la Convention de Genève "38.

Dans un premier temps, les représentants des États s'étaient contentés d'échanger leurs positions au sein d'un forum, le C.I.R.E.A. ${ }^{39}$. Francis Lott, alors Directeur de l'O.F.P.R.A., s'était félicité en janvier $1996^{40}$ des travaux du " club des directeurs des O.F.P.R.A. des Quinze " qui travaille à " rapprocher nos façons de faire ". En outre, il citait deux exemples de coopération bilatérale : pour les Algériens, " nous avons réussi à faire en sorte que nos collègues allemands appliquent notre politique " et pour les SriLankais, " inversement, nous avons rapproché nos chiffres des leurs ". Francis Lott concluait que " les chances deviennent à peu près les mêmes partout ", c'est-à-dire presque nulles; en 1995 : taux de reconnaissance pour les Algériens 1\%, pour les SriLankais environ $5 \%$ (hors principe de l'unité de famille).

Des pays " sûrs " ?

Prévue par certaines législations nationales, la notion de pays d'origine " sûr " (ou " pays dans lesquels il n'existe généralement pas de risque sérieux de persécution ") a pour but de traiter les demandes de certains pays dans le cadre des procédures accélérées, voire de les exclure automatiquement des procédures et de leur refuser ainsi toute possibilité d'obtenir un statut de réfugié ${ }^{41}$.

L'O.F.P.R.A. n'applique pas formellement la notion de pays " sûr ", néanmoins, les ressortissants de certaines nationalités éprouvent bien des difficultés à obtenir le statut. À propos des Zaïrois, Francis Lott, en janvier 1996, précisait à la commission 
parlementaire qu'" avant 1989 , nous étions un peu généreux, nous sommes plus exigeants depuis quelques années [...] une mission de l'O.F.P.R.A. [...] a abouti à un rapport dont nous appliquons les conclusions de manière très rigoureuse ". À propos de deux Ivoiriens reconnus réfugiés par la Commission des recours, il écrivait en 1991 à l'ambassadeur de France à Abidjan qu'il " avait appris avec regret qu'il devait établir des statuts de réfugié " et qu'il " estimait naturellement que la situation de la Côte d'Ivoire ne justifie en aucune mesure que la France accorde l'asile à ces ressortissants ".

En cas de changements fondamentaux dans le régime politique d'un pays, les ressortissants de ce pays peuvent ne plus se voir renouveler leur certificat de réfugié, en application de la "clause de cessation " de la Convention de Genève ${ }^{42}$. Il arrive que l'O.F.P.R.A. invoque cette clause pour un pays avant que la situation ne s'y soit stabilisée. Ce fut le cas pour la Roumanie en juin 1995 alors que le H.C.R. demandait qu'elle ne soit pas invoquée avant que " certains aspects restrictifs de la législation et la pratique, notamment sur les droits de quelques minorités et groupes sociaux, ne puissent être modifiés permettant aux groupes de bénéficier de leurs droits sans discrimination ou entrave ". En outre, l'O.F.P.R.A. précisait que " les demandes d'admission au statut ne peuvent, désormais, être accueillies favorablement " qu'en cas de " circonstances exceptionnelles ". Cette affirmation laissait entendre que les Roumains arrivant après cette décision n'ont pratiquement aucun droit à un examen attentif. Francis Lott, alors directeur de l'Office, a même souhaité que, à l'avenir, " la loi permette d'exclure les demandeurs dont la nationalité a été l'objet d'une décision de cessation ${ }^{443}$.

60 Le déroulement de la procédure

61 La procédure aboutit à une décision de reconnaissance du statut de réfugié ou de rejet de la demande par l'O.F.P.R.A. Outre la procédure prioritaire (voir Une procédure prioritaire), l'Office a défini deux procédures d'instruction distinctes, les procédures courte et longue. Le choix de l'une ou l'autre est lié à l'analyse de la situation régnant dans le pays d'origine et du contenu de la demande.

Des procédures plus ou moins équitables

63

- La procédure courte d'instruction simplifiée s'applique, selon l'O.F.P.R.A., aux demandes considérées comme manifestement infondées ${ }^{44}$ : " lorsqu'elle émane d'un ressortissant d'un pays où l'on ne dénonce plus de violations graves de droits de l'homme, lorsque le récit ne fait pas apparaître d'élément circonstancié ou personnalisé, lorsque la demande provient d'un pays où les changements dans la nature du régime ont conduit à appliquer une clause de cessation du statut de réfugié, lorsque la demande ne se fonde pas sur les motifs de la Convention de Genève, lorsqu'une fraude a été décelée ou que le dossier est incomplet ". Elle implique en général l'absence d'entretien individuel avec le requérant.

64

- La procédure longue s'applique dans les autres cas et, notamment, dès lors que le récit est circonstancié et personnalisé, riche en détails ou accompagné de documents appuyant les allégations de l'intéressé. Elle implique souvent un entretien individuel avec le requérant qui a pour objet d'établir la vraisemblance des faits invoqués mais aussi la façon dont le requérant a quitté son pays d'origine et l'itinéraire qu'il a emprunté. 
- L'examen par priorité : lorsqu'une préfecture juge que la procédure est utilisée de manière abusive, notamment pour faire échec à une mesure d'éloignement du territoire (ordonnance du 2 novembre 1945, article 31 bis- 4), l'office examine les demandes " par priorité ", souvent sans entretien. Cette procédure appliquée à 516 demandes en 1994, à 620 en 1995 et à 581 en 1996 peut concerner des étrangers qui viennent d'arriver sur le territoire français et sont contrôlés avant même d'avoir pu déposer leur demande d'asile. Ces demandeurs n'auront alors pas droit à un examen complet de leur dossier : il arrive aussi qu'une convocation leur soit adressée au centre de rétention alors qu'ils l'ont déjà quitté. ministère des Affaires étrangères a rappelé qu'une mission d'audit avait recommandé l'audition systématique des demandeurs par l'office et il faisait d'ailleurs remarquer que " l'entretien apparaît comme la méthode la plus sûre pour reconnaître des déclarations authentiques par opposition aux récits 'clef en main' ". Le président de la Commission des recours les considérait même " indispensables [...] lorsqu'on a affaire à des dossiers incertains ou des récits stéréotypés ". Malheureusement, l'O.F.P.R.A. considère que " les fraudeurs, les utilisateurs de filières n'ont aucune raison d'être entendus ", pas plus que les demandeurs ayant adressé des " dossiers manifestement fondés ou infondés ".

71 S'ils ont la chance d'être convoqués, certains demandeurs peuvent néanmoins éprouver des difficultés pour s'y rendre. Notamment financières, s'ils résident en province ${ }^{46}$. L'O.F.P.R.A. rencontre un problème autrement plus compliqué avec ceux qui résident 
dans les départements et territoires d'outre-mer. Les demandes d'asile ne sont en fait pas aisées à faire enregistrer dans les D.O.M.-T.O.M. et, lorsqu'elles le sont, l'Office ne peut sérieusement proposer à un requérant de se rendre à son siège pour un entretien. En 1995, la préfecture de Guyane avait finalement enregistré quelques demandes de ressortissants haïtiens et l'O.F.P.R.A. avait envoyé une délégation sur place pour procéder à des auditions.

On peut également regretter qu'aucun document consignant les échanges entre l'O.F.P.R.A. et le demandeur pendant l'audition ne soit communiqué à ce dernier. En cas de rejet ultérieur, cela lui permettrait de connaître les raisons profondes de la décision et de mieux préparer son recours devant la Commission des recours. ... sans droit à un accompagnement de soutien

Il est regrettable que l'O.F.P.R.A. refuse que les demandeurs soient accompagnés, lors de l'entretien, par un ami, un membre d'une association ou un avocat. Comme l'office a le statut d'établissement public et non de juridiction, la procédure n'est en effet ni contradictoire, ni publique. Le directeur de l'office n'accepte, éventuellement, qu'un entretien " séparé " avec un tiers ; il juge en effet que la spontanéité du demandeur est essentielle et que celle-ci exclut la présence d'un tiers. En outre, le candidat réfugié n'étant pas dans la situation d'une personne ayant à se défendre, un avocat ne peut être entendu qu'au cours d'un entretien séparé.

Pourtant, le demandeur peut être impressionné et la présence d'une personne connaissant son dossier peut permettre d'expliquer des questions mal comprises, de repréciser des réponses mal formulées ou d'éviter des malentendus. Le Guide du H.C.R. (\$ 198 et 200) précise par exemple qu'une personne " qui a appris à craindre les autorités de son pays peut continuer à éprouver de la défiance à l'égard de toute autre autorité [...] et craindre de parler librement [...] l'examinateur devra mettre le demandeur en confiance ". L'accompagnement d'une personne de confiance peut certainement y aider.

\section{NOTES}

1. L'article 31 bis 2 de l'ordonnance de 1945 , permettant au préfet de refuser l'admission au séjour en cas d'admissibilité et de protection effective dans un autre État, semble rarement utilisé, parce que compliqué à mettre en œuvre.

2. Selon la circulaire du 8 février 1994 : fraude délibérée : demandes multiples sous plusieurs identités, simultanées dans le but de cumuler le bénéfice d'allocations ou successives dans le but de se maintenir en France après un rejet définitif et recours abusif : demande de réexamen faite à l'O.F.P.R.A. après un premier rejet définitif dénuée de tout élément nouveau. Selon la loi du 23 avril 1997, " constitue en particulier un recours abusif, la présentation frauduleuse de plusieurs demandes sous des identités différentes ".

3. Soit 2\% de l'ensemble des demandes traitées en 1994, 3\% en 1995 et 1996. 
4. La CIMADE note que, sur les 115 demandes déposées en urgence au centre de rétention de Nice en 1996, 42 ont fait l'objet d'une convocation par l'O.F.P.R.A. pour audition. Pour 3 autres demandes, l'O.F.P.R.A. a demandé à la préfecture de délivrer une A.P.S. et d'appliquer la procédure normale de demande d'asile.

5. Dans le même sens, le tribunal administratif de Grenoble, statuant sur un recours contre un arrêté préfectoral de reconduite frontière, a considéré que, " si la demande n'a été formée que lors de l'interpellation [...] il ne résulte pas des pièces du dossier qu'elle ait eu manifestement pour seul objet de faire échec à une décision de reconduite à la frontière " (T.A., 8 décembre 1994). Le Conseil d'État confirme cette interprétation en jugeant " que les seules circonstances que M. L soit entré en France après avoir traversé [...] la Pologne et l'Allemagne et n'ait sollicité la demande de reconnaissance de statut de réfugié que le jour de son interpellation, 48 heures après son arrivée sur le territoire français, ne sont pas de nature à établir que ladite demande ait eu manifestement pour seul objet de faire échec, dans un but dilatoire, à la mesure de reconduite " (C.E., 3 mars 1997).

6. Dans cette affaire, le Préfet reviendra sur cette décision et lui délivrera un titre de séjour mais sans admettre explicitement que l'appréciation du caractère abusif de la demande avait été abusive. Il se contentera de dire que " les conditions d'obtention de son passeport et de son visa, n'avaient pas été parfaitement éclaircies " (lettre du 22 octobre 1996). Or, comme l'a rappelé le Conseil d'État dans une autre affaire, "La circonstance que M. B aurait eu recours, pour obtenir le visa de 30 jours qui lui a permis d'entrer en France, à une manoeuvre de caractère frauduleux, ne suffit pas à établir que la demande d'asile repose sur une fraude délibérée au sens de l'article 31 bis 4 " (C.E., 2 octobre 1996). Dans ses conclusions, le commissaire du gouvernement avait même précisé, qu'" on ne doit pas exclure que des personnes effectivement persécutées aient eu à recourir à de tels subterfuges pour quitter leur pays ".

7. En août 1995, un enfant de sept ans avait été tué par un policier de la DICCILEC, lors d'un contrôle nocturne près de la frontière italienne.

8. C.E., 2 juin 1997.

9. Propos tirés de la lettre de la Direction des libertés publiques et des affaires juridiques (D.L.P.A.J.) du ministère de l'Intérieur en date du 4 juillet 1996.

10. Circulaire du 8 février 1994.

11. Article 32 bis de l'ordonnance de 1945.

12. Idem.

13. Dans un arrêt du 27 mars 1996 la Cour de cassation a jugé, à propos du recours d'un ressortissant sénégalais contre l'ordonnance de prolongation du délai de rétention, " qu'ayant constaté que M. G. ne parlait pas français et n'avait pas été assisté d'un interprète lors de son audition par les services de police, et s'agissant de la protection des libertés individuelles [...] l'audition [...] et la procédure judiciaire [...] étaient nulles ". L'article 35 bis alinéa 3 précise que, lorsqu'il est mis en rétention, "l'étranger est immédiatement informé de ses droits par l'intermédiaire d'un interprète s'il ne connaît pas la langue française".

14. article 22 bis I de l'ordonnance de 1945.

15. Lettre du directeur de l'O.F.P.R.A. du 7 février 1995.

16. Compte rendu de la réunion du 29 mai 1995 du Conseil de l'O.F.P.R.A.

17. Compte rendu de la réunion du 4 août 1995 du Conseil de l'O.F.P.R.A.

18. Ou pour l'étranger muni d'un visa de longue durée, 6 mois avec droit au travail. 
19. voir infra Les auteurs de persécution. Ces formes de protection sont appelées " protection temporaire ", " asile territorial ", " exceptional leave to remain " par le Royaume-Uni, " protection collective " en Norvège...

20. Un télégramme du ministère de l'Intérieur aux préfets, en date du 23 décembre 1996, déclare que la situation qui prévaut dans les États de l'ex-Yougoslavie " rend désormais possible (...) la mise à exécution des mesures d'éloignement des personnes originaires de la République de Bosnie-Herzégovine et de la République fédérale de Yougoslavie ".

21. Voir infra Les régularisations exceptionnelles.

22. Jusqu'en 1991, le nombre des demandes de reconnaissance du statut de réfugié émanant des ressortissants algériens était inférieur à 200 ; 619 demandes ont été déposées en 1992, 1099 en 1993, 2303 en 1994, 1800 en 1995 et 643 en 1996.

23. Voir infra Détermination du statut de réfugié, une jurisprudence restrictive.

24. Lors d'une intervention à l'Assemblée nationale le 19 août 1996, le ministre de l'Intérieur a déclaré que " cette procédure individuelle est régulièrement utilisée ".

25. Le nombre de visas délivrés aux Algériens, toutes catégories confondues, a chuté de manière très importante ces dernières années : il est passé de 800000 en 1989 à environ 40000 en 1996.

26. Cette situation a été modifiée par le télégramme du 23 décembre 1996 précité : l'exécution des mesures d'éloignement déjà prises est à nouveau possible, l'A.P.S. sera renouvelée aux personnes qui en bénéficiaient mais ne sera pas délivrée aux nouveaux arrivants.

27. Les Albanais du Kosovo ainsi que d'autres nationalités de l'ex-Yougoslavie se voient refuser les A.P.S., sous prétexte que " La situation du Kosovo ne peut être assimilée à celle d'autres régions [...] considérées comme des zones troublées au sens des instructions ministérielles [précitées] ".

28. Télex du 14 septembre 1992.

29. Note introductive à la réunion informelle sur la protection temporaire organisée par le H.C.R. le 21 janvier 1993 à Genève.

30. Lettre de la Direction des libertés publiques et des affaires juridiques, 26 mai 1994.

31. Dans un document qui s'intitule Solutions à l'issue conflit-Programme du H.C.R. en Bosnie-Herzégovine et dans les autres pays de la région.

32. Notamment celle des réfugiés qui risquent de se retrouver dans des régions dans lesquelles ils sont en minorité ethnique. Cela ne signifie pas qu'il soit satisfaisant en l'absence de danger de les renvoyer dans des régions qui ne sont pas leur région d'origine, les mettant de fait en situation de personnes déplacées.

33. L'Allemagne n'a pas suivi ces recommandations en décidant de lever officiellement la protection temporaire le 1er juillet 1996.

34. Bosnie-Herzégovine, Croatie, République fédérative de Yougoslavie (Serbie et Monténégro).

35. Il faut entendre par "mariage mixte" le mariage entre des personnes ressortissantes de l'ex-Yougoslavie mais n'appartenant pas à la même communauté.

36. Le S.S.A.E. : Service social d'aide aux émigrants.

37. Le paragraphe 190 du Guide du H.C.R. prévoit que " la demande doit être examinée [...] par un personnel qualifié, ayant les connaissances et l'expérience voulues et capable de comprendre les besoins particuliers du demandeur ". Depuis la restructuration de 1994, il peut être demandé aux O.P. $d$ '" instruire les demandes 
d'autres secteurs par rapport à leurs actuelles attributions ". Il ne faudrait pas que cela nuise à la spécialisation indispensable que requiert un traitement sérieux des dossiers. 38. Communiqué du H.C.R., Le H.C.R. exprime des réserves sur la politique de l'asile en Europe, 24 novembre 1995.

39. Centre d'information, de recherche et d'échanges sur l'asile, voir Le Traité de Maastricht et l'Union européenne.

40. Audition, le 30 janvier 1996, par la Commission d'enquête sur l'immigration clandestine et le séjour irrégulier d'étrangers en France de l'Assemblée nationale. 41. Le H.C.R. a souligné que ceci constituerait une réserve de fait à l'article 1 . A-2 de la Convention de 1951. Or l'article 42 interdit toutes réserves à l'article 1 . De plus, une telle attitude pourrait être contraire aux dispositions de l'article 3 de la Convention de 1951, qui interdit les discriminations quant au pays d'origine.

42. Ce fut le cas pour des pays comme le Chili, le Cap-Vert, le Bénin, la Pologne, la Hongrie. Cette décision est susceptible d'un recours. L'étranger à qui la qualité de réfugié est retirée conserve en général sa carte de résident, récupère un passeport de son pays et peut y retourner rendre visite à sa famille et à ses amis. Les ressortissants de ces pays arrivant après l'application de cette clause ont toujours le droit de solliciter la reconnaissance du statut de réfugié mais ont très peu de chances de l'obtenir.

43. Janvier 1996, audition par la Commission parlementaire d'enquête sur l'immigration clandestine et le séjour irrégulier d'étrangers en France.

44. D'après l'O.F.P.R.A., cette procédure s'applique aussi aux demandes manifestement fondées : cas des demandeurs ayant un visa d'établissement, étant connus pour leur action, provenant d'un pays où " les craintes de persécutions sont objectivées par la gravité de la situation " ou étant admis " sur quota ".

45. Selon le directeur de l'O.F.P.R.A., lors d'une rencontre en septembre 1996 avec des associations : Tamouls du Sri-Lanka, Sikhs d'Inde, Bangladais, Somaliens, Ethiopiens, Mauritaniens, Angolais, Burundais, Turcs, Algériens sont systématiquement convoqués. 46. Une majorité des demandeurs vivent dans la région parisienne. L'installation d'antennes locales de l'office dans certains lieux du territoire où des problèmes spécifiques se posent a été envisagée mais actuellement les demandeurs doivent se déplacer au siège.

\section{INDEX}

Mots-clés : asile, droit 